\title{
A rare problem in a pregnant woman with COVID-19 pneumonia: Pneumomediastinum and subcutaneous emphysema
}

\author{
Esra Sultan Karabulut Keklik¹(D, Hakan Dal' ${ }^{1}$, Şahin Bozok² ${ }^{2}$ \\ ${ }^{1}$ Department of Anesthesiology and Reanimation, Uşak University, Training and Research Hospital, Uşak, Turkey
}

${ }^{2}$ Department of Cardiovascular Surgery, Uşak University Faculty of Medicine, Uşak, Turkey

Received: July 06, 2020 Accepted: October 27, 2020 Published online: November 13, 2020

\begin{abstract}
Novel coronavirus disease-2019 (COVID-19) has affected millions of individuals within in a short period of time, resulting in hundreds of thousands of deaths, and has led to a crisis worldwide. A 22-year-old pregnant woman (32 weeks of gestation) was admitted to our clinic with a suspicion of COVID-19. Initially, radiological evaluation using computed tomography (CT) imaging was unable to be performed, as the patient refused imaging study; however, we were able to obtain CT following emergency cesarean section. On CT scan, pulmonary lesions were predominant at the lower zones and progressed to confluent bilateral consolidation with pneumomediastinum, confirming COVID-19 pneumonia and pneumomediastinum complications. In conclusion, although rare, spontaneous pneumomediastinum should be considered in COVID-19 infection.
\end{abstract}

Keywords: Complication, computed tomography, coronavirus, COVID-19, pneumomediastinum, pneumonia, pregnant woman.

Pneumomediastinum is classified as primary (spontaneous) or secondary pneumomediastinum..$^{[1,2]}$ There is no obvious underlying cause in spontaneous mediastinum, while there is an apparent cause such as trauma, intra-thoracic infection, and injuries of respiratory or gastrointestinal tracts in the secondary type. Majority of cases develop due to traumatic causes. ${ }^{[1,2]}$ Although spontaneous pneumomediastinum is rare, it is often seen in healthy, young men as a result of rupture of the peripheral pulmonary alveoli. $^{[1,2]}$ Other potential causes include barotrauma during mechanical ventilation, hyperbaric therapy, elevation phase of diving, asthma or obstructive airway, such as foreign body aspiration. In addition, pneumomediastinum has been also reported following tooth extraction, tonsillectomy, tracheostomy, head and neck surgery, and craniofacial trauma..$^{[1,2]}$

In late December 2019, several pneumonia cases with an unknown etiology were reported from Wuhan Province of China and evolved to global pandemics. The causative agent was initially designated as acute respiratory distress syndrome-coronavirus-2 (SARS-CoV-2); subsequently, it was denoted as novel coronavirus disease-2019 by the World Health Organization (WHO). ${ }^{[3,4]}$ Pulmonary parenchymal opacities are frequently seen on chest radiographs.
Complications of severe COVID-19 pneumonia were reported in few cases. ${ }^{[5]}$

In this article, we report a young pregnant woman with pneumomediastinum as a potential, rare complication of COVID-19 pneumonia.

\section{CASE REPORT}

A 22-year-old pregnant woman (32 weeks of gestation) without a known systemic disease was admitted to our clinic with fever. The patient refused computed tomography (CT) imaging due to potential harms of CT during pregnancy. She had no respiratory, circulatory, or metabolic problem requiring intensive care unit (ICU) admission and was admitted to the pandemic clinic with suspected COVID-19. As the polymerase chain reaction (PCR) test was positive, a loading dose of hydroxychloroquine $400 \mathrm{mg}$ b.i.d.,

Corresponding author: Şahin Bozok, MD. Uşak Üniversitesi Tip Fakültesi Kalp ve Damar Cerrahisi Anabilim Dalı, 64000 Uşak, Türkiye.

Tel: +90 276 - 2240000 e-mail: sahinboz@yahoo.com

Citation:

Karabulut Keklik ES, Dal H, Bozok Ș. A rare problem in a pregnant woman with COVID-19 pneumonia: Pneumomediastinum and subcutaneous emphysema. Cardiovasc Surg Int 2020;7(3):186-189. 
followed by a maintenance dose of $200 \mathrm{mg}$ b.i.d. and a loading dose of azithromycin $500 \mathrm{mg}$ b.i.d., followed by a maintenance dose of $250 \mathrm{mg}$ b.i.d. were prescribed based on the COVID-19 National Treatment Guidelines. On Day 3 after admission, the patient had hypoxemia (peripheral oxygen saturation $\left[\mathrm{sPO}_{2}\right]: 80 \%$ ) and tachypnea (respiration rate: $36 / \mathrm{min}$ ); thus, she was admitted to the pandemic ICU.

In the first assessment in the ICU, the patient had dyspnea, severe tachypnea (respiration rate: 36/min) and tachycardia (heart rate: $130 \mathrm{bpm}$ ). The $\mathrm{sPO}_{2}$ value was $78 \%$ under oxygen supplementation (6 to $8 \mathrm{~L} / \mathrm{min}$ ) via the face mask. Non-invasive mechanical ventilation was initiated (via full-face mask; pressure-assisted ventilation mode; positive end expiratory pressure [PEEP]: $7 \mathrm{cmH}_{2} \mathrm{O}$ and pressure support [PS]: $14 \mathrm{cmH}_{2} \mathrm{O} ; 50 \%$ oxygen supplementation). The $\mathrm{sPO}_{2}$ value was improved to $97 \%$ and tachypnea was decreased (respiration rate: $24 / \mathrm{min}$ ). After ICU admission, high-flow nasal oxygen supplementation $\left(37^{\circ} \mathrm{C} ; 60 \mathrm{~L} / \mathrm{min}\right.$ flow and $40 \%$ oxygen) and interrupted non-invasive mechanical ventilation $(50 \%$ oxygen; PEEP: $7 \mathrm{cmH}_{2} \mathrm{O}$; PS: $14 \mathrm{cmH}_{2} \mathrm{O}$; pressure-assisted ventilation mode, via an oronasal mask) were applied until Day 9.

On Day 4 after the ICU admission, new-onset chest pain developed. Subcutaneous emphysema was palpated on the upper one-third of left thoracic wall, particularly over the clavicle. Chest auscultation findings were normal. The radiological chest imaging was planned to the patient; however, the patient refused any type of imaging study. Thus, lung sonography was planned to the patient. However, due to the settings of COVID-19 ICU and unfavorable general health status of the patient, an adequate assessment could not be performed. As there was no alteration in clinical findings, hypoxemia level and ventilation parameters, we continued to follow the patient with the available status.

The patient was maintained by alternate ventilation strategy (5-h high-flow nasal oxygen followed by 1-h non-invasive mechanical ventilation; however, on Day 7, the need for non-invasive mechanical ventilation increased due to hypoxemia with a $\mathrm{SPO}_{2}$ of 85 to $90 \mathrm{cmH}_{2} \mathrm{O}$ ); thus, a sonography was performed by an obstetrician, revealing the decreased amnion fluid. An emergency cesarean section was performed
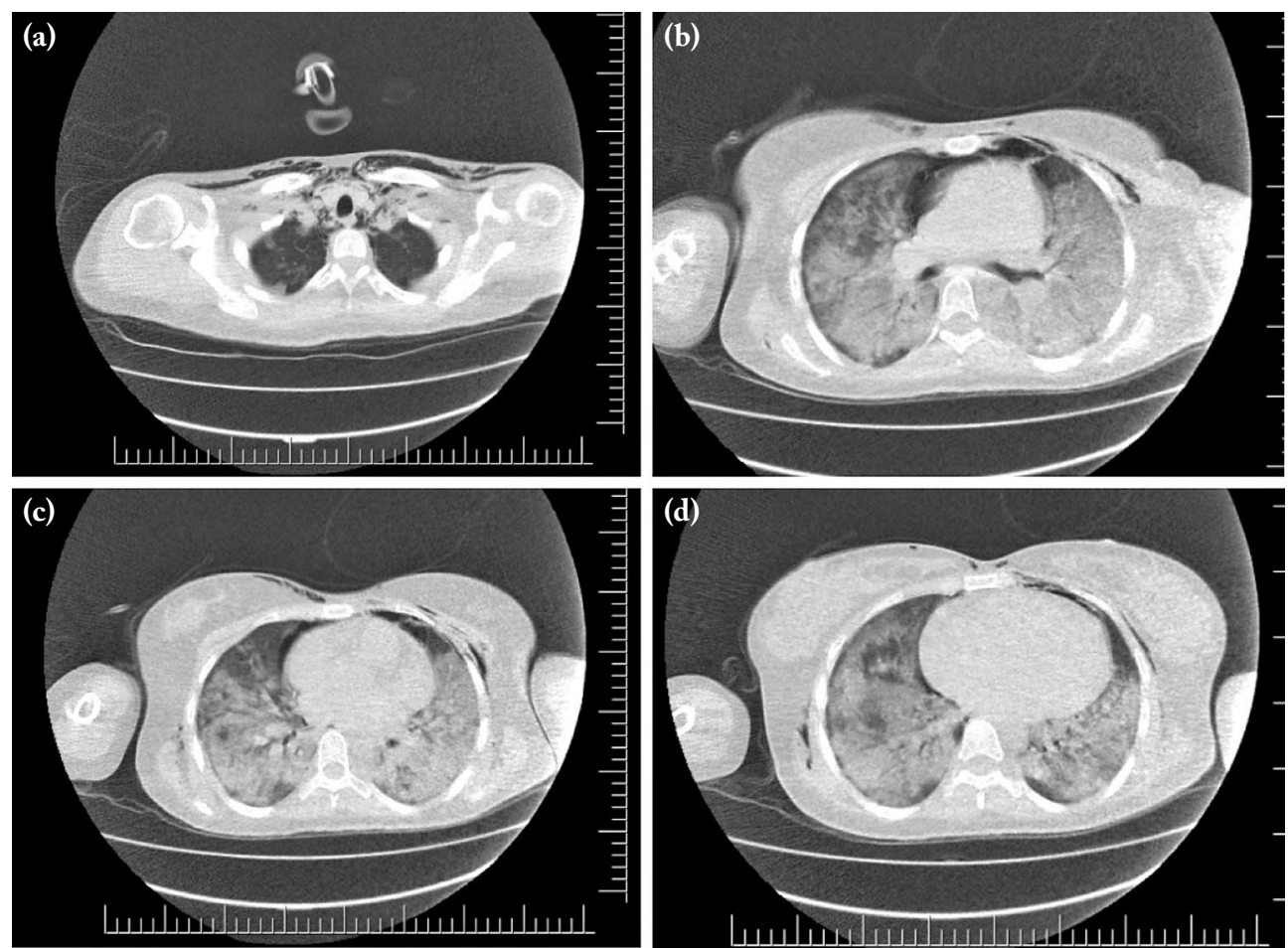

Figure 1. Initial computed tomography scans after cesarean section. (a) Subcutaneous emphysema; (b-d) pneumomediastinum and bilateral ground-glass opacities. 
under spinal due to the decreased amnion fluid and profound hypoxemia anesthesia in the patient on non-invasive mechanical ventilation. Two PCR tests with a 24-h interval were negative for COVID-19 in the infant; thus, newborn was discharged after seven days of ICU admission.

The CT imaging which was refused initially by the patient was performed after cesarean section and revealed multiple diffuse patchy consolidation areas and ground-glass opacities in both lungs and pneumomediastinum, confirming COVID-19 pneumonia, pneumomediastinum without pneumothorax and subcutaneous emphysema (Figure 1a-d). As pneumothorax was lacking and pneumomediastinum caused no compression, no surgery was planned. On Day 2 after cesarean section, orotracheal intubation was performed and mechanical ventilation was initiated due to worsening in the general health status and respiratory parameters on Day 9 after admission. The following parameters were used for ventilation: pressure-regulated volume control mode; tidal volume, $550 \mathrm{~mL}$, frequency, 16/min, PEEP: $10 \mathrm{cmH}_{2} \mathrm{O}$ and $\mathrm{FiO}_{2}: 80 \%$.
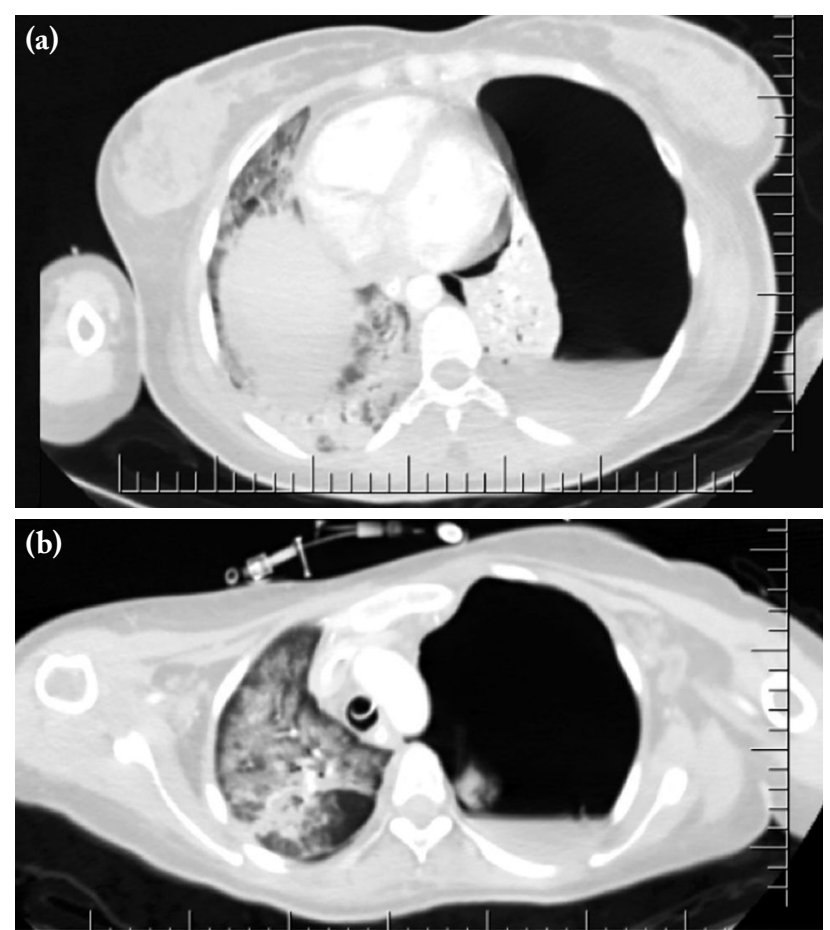

Figure 2. Control computed tomography scans showing no pneumomediastinum. (a) Massive pneumothorax and pleural effusion at left; (b) right-sided cardiac shift and massive pneumothorax at left.
Due to worsened gas exchange (P/F:70), the patient was placed to the prolonged prone position $(22 \mathrm{~h})$. On Day 14 after detection of pneumomediastinum on the first CT scan, a control CT imaging was performed in the patient who had deterioration in gas exchange, respiratory parameters, and hemodynamics and revealed massive pneumothorax in the left lung and disappearance of previous subcutaneous emphysema (Figure 2a, b). Thus, a chest tube was inserted via thoracostomy from the left side. During follow-up, Acinetobacter baumannii sepsis, deep venous thrombosis in the left femoral vein, and subsequent pulmonary embolism developed. The patient died on Day 22 after the ICU admission, despite all efforts.

\section{DISCUSSION}

Pneumomediastinum is a rare entity. In previous studies, the pneumomediastinum incidence has been reported as $1: 800$ to $1: 42,000$ in children presented to emergency department and 1:15,500 admissions in general population. The mean age at onset is 19 years. ${ }^{[1,2]}$ The pain is the most common presenting symptom in 80 to $90 \%$ of patients. ${ }^{[1,2]}$ In our case, similarly, the first complaint in the ICU was chest pain.

Computed tomography plays a major role in the diagnosis of COVID-19 pneumonia, which is an ongoing global problem. Although many parenchymal or non-parenchymal findings are seen on CT scans, the most common finding is ground-glass opacities in parenchyma. ${ }^{[6]}$ Spontaneous pneumomediastinum is an extremely rare condition. The most common clinical findings include fever, cough, myalgia, or fatigue. ${ }^{[6]}$ Our patient refused radiological evaluation due to potential harms of CT during pregnancy; thus, the diagnosis was made based on PCR testing and clinical presentation, and no radiological examination was able to be performed initially. Such findings should be addressed meticulously, particularly in the course of COVID-19 pneumonia. Thus, diagnosis through early imaging studies and timely treatment of COVID-19 complications can improve therapeutic effectiveness and decrease mortality. ${ }^{[5]}$

Pneumothorax and pneumomediastinum have been rarely reported in COVID-19 pneumonia. The pneumomediastinum was not only observed without parenchymal lesion, ${ }^{[7]}$ but also observed with ground-glass opacities, findings of pneumonia, and pulmonary parenchymal injury. ${ }^{[5,8]}$ In our patient, pneumomediastinum was present with findings 
of COVID-19 pneumonia. In previous reports, there was no comorbid condition such as asthma or chronic lung disease which may contribute to available condition or invasive interventions leading to iatrogenic pneumothorax; therefore, all previous cases were considered COVID-19-related spontaneous pneumomediastinum..$^{[5,7,8]}$ Although pregnancy, itself, has been reported as a cause of spontaneous mediastinum, ${ }^{[1,2]}$ we believe that severe, dry cough, and pulmonary injury caused by COVID-19 could be the cause of pneumomediastinum. Although severe pulmonary injury was seen in on the initial thoracic CT scan, it was thought that pneumomediastinum without pneumothorax was surprising.

During pandemic, four pregnant women were treated in our hospital. All patients were treated in accordance with the COVID-19 National Treatment Guidelines. Of these patients, three women were discharged successfully; however, the present case died.

In conclusion, although spontaneous pneumomediastinum is rarely seen in COVID-19 infection, it can develop due to severe cough and respiratory distress. Spontaneous pneumomediastinum is usually a self-limiting disorder, although the exact mechanism is still unknown. However, it has potential to cause severe circulatory or respiratory disorder. Thus, spontaneous pneumomediastinum in COVID-19 patients should be closely monitored as a potential marker for progression.

\section{Declaration of conflicting interests}

The authors declared no conflicts of interest with respect to the authorship and/or publication of this article.

\section{Funding}

The authors received no financial support for the research and/or authorship of this article.

\section{REFERENCES}

1. Ichinose J, Nagayama K, Hino H, Nitadori J, Anraku M, Murakawa T, et al. Results of surgical treatment for secondary spontaneous pneumothorax according to underlying diseases. Eur J Cardiothorac Surg 2016;49:1132-6.

2. Işık H, Sapmaz E, Özbey $M$, Çaylak H, Şengül İnan M. A rare cause of pneumomediastinum and subcutaneous emphysema: Tooth extraction. Turk Gogus Kalp Dama 2020;28:390-3.

3. Yilmaz Y, Kamer E. Planning and safety of surgical operation in COVID-19 Pandemic. Ege Klin Tip Derg 2020;58Supp:55-61.

4. Kamer E, Çolak T. What to do when a patient infected with COVID-19 needs an operation: A pre-surgery, perisurgery and post-surgery guide. Turk J Colorectal Dis 2020;30:1-8.

5. Sun R, Liu H, Wang X. Mediastinal Emphysema, Giant Bulla, and Pneumothorax Developed during the Course of COVID-19 Pneumonia. Korean J Radiol 2020;21:541-4.

6. Zu ZY, Jiang MD, Xu PP, Chen W, Ni QQ, Lu GM, et al. Coronavirus Disease 2019 (COVID-19): A Perspective from China. Radiology 2020;296:E15-E25.

7. Kolani S, Houari N, Haloua M, Alaoui Lamrani Y, Boubbou $M$, Serraj $M$, et al. Spontaneous pneumomediastinum occurring in the SARS-COV-2 infection. IDCases 2020;21:e00806.

8. Wang J, Su X, Zhang T, Zheng C. Spontaneous Pneumomediastinum: A Probable Unusual Complication of Coronavirus Disease 2019 (COVID-19) Pneumonia. Korean J Radiol 2020;21:627-8. 